\title{
ACTIVIDAD INSECTICIDA DEL ACEITE ESENCIAL DEL PAICO Chenopodium ambrosioides L. SOBRE Sitophilus zeamais Motschulsky
}

\section{INSECTICIDAL ACTIVITY OF ESSENTIAL OIL OF Chenopodium ambrosioides L. AGAINST Sitophilus zeamais Motschulsky}

Julia Aros ${ }^{1}$, Gonzalo Silva-Aguayo ${ }^{1 *}$, Susana Fischer ${ }^{1}$, Inés Figueroa ${ }^{1}$, J. Concepción RodríguezMaciel $^{2}$, Angel Lagunes-Tejeda ${ }^{2}$, Gloria S. Castañeda-Ramírez ${ }^{3}$, y Liliana Aguilar-Marcelino ${ }^{3}$

${ }^{1}$ Departamento de Producción Vegetal, Facultad de Agronomía, Universidad de Concepción, Vicente Méndez 595, Chillán, Chile.

${ }^{2}$ Programa de Entomología y Acarología, Colegio de Postgraduados, Km 36,5 Carretera Federal, México-Texcoco, Montecillo, Estado de México, México.

${ }^{3}$ Centro Nacional de Investigación Disciplinaria en Salud Animal e Inocuidad, INIFAP, Km 11 Carretera Federal Cuernavaca-Cuautla, Jiutepec, Estado de Morelos, México.

* Autor para correspondencia E-mail: gosilva@udec.cl

\section{RESUMEN}

Sitophilus zeamais Motschulsky es a nivel mundial una de las principales plagas de los cereales almacenados y su control se basa en insecticidas sintéticos, pero su uso irracional ha provocado contaminación del ambiente y resistencia. Los aceites esenciales han mostrado efecto insecticida de contacto y fumigante por lo que podrían constituir una alternativa a los compuestos sintéticos. Por este motivo se evaluaron, en condiciones de laboratorio, las propiedades insecticidas del aceite esencial de Chenopodium ambrosioides L. contra adultos de $S$. zeamais. Las variables evaluadas fueron toxicidad por contacto y fumigación, y actividad antialimentaria y repelente. La mortalidad por contacto no superó el $30 \%$ en todos los tratamientos evaluados. La mayor reducción de la $\mathrm{F}_{1}$ se observó en el tratamiento de $4,0 \%$ con $41,3 \%$ de emergencia $(58,7 \%$ de inhibición). La pérdida de peso de los granos fue menor a 9,0\% y la germinación del maíz no se vio afectada. Todos los tratamientos mostraron actividad fumigante y las concentraciones iguales o superiores a $0,5 \%$ tuvieron efecto repelente y antialimentario. Se concluye que el aceite esencial de Ch. ambrosioides presenta potencial para el control de S. zeamais.

Palabras clave: gorgojo del maíz, Sitophilus zeamais, granos almacenados, paico, Chenopodium ambrosioides, insecticida vegetal.

\section{ABSTRACT}

Sitophilus zeamais is one of the major pests of stored cereals worldwide and its control is based on the use of synthetic insecticides, but their irrational use has caused environmental pollution and resistance. Essential oils have shown contact and fumigant insecticidal effect and could be an alternative to synthetic compounds. Hence, the insecticidal properties of essential oil of Chenopodium ambrosioides L. were assessed under laboratory conditions against adults of Sitophilus. zeamais. The variables studied were contact and fumigant toxicity, and antifeedant and repellent activity. Mortality by contact toxicity did not exceed $30 \%$ in all the treatments evaluated. The highest $F_{1}$ reduction was observed in the treatment of $4.0 \%$ with $41.3 \%$ of emergence $(58.7 \%$ of inhibition). Grain weight loss 
was lower than $9.0 \%$ and maize germination was not affected. All treatments exhibited fumigant activity, while concentrations equal to or lower than $0.5 \%$ showed repellent and feeding deterrent effect. It can be concluded that essential oil of $C h$. ambrosioides has potential for S. zeamais control.

Key words: Maize weevil, Sitophilus zeamais, stored grains, Mexican tea, Chenopodium ambrosioides, botanical insecticides.

\section{INTRODUCCIÓN}

El gorgojo del maíz (Sitophilus zeamais Mostchulsky; Coleoptera: Curculionidae) es una de las principales plagas de los cereales almacenados a nivel mundial (Danho et al., 2002; Tavares y Vendramim, 2005; Suthisut et al., 2011). Actualmente los insecticidas sintéticos son el método más utilizado para la protección de semillas contra plagas de productos almacenados (Isman, 2015). Sin embargo, su uso irracional ha provocado problemas como contaminación del ambiente, alta toxicidad residual y la resistencia de los insectos (Sarwar, 2015). En este contexto, los aceites esenciales representan una alternativa para el control de plagas asociadas a granos almacenados (Isman, 2015).

El paico (Chenopodium ambrosioides L.; Chenopodiaceae) es una planta aromática y medicinal que se ha utilizado por siglos como condimento y contra parásitos intestinales (Tavares y Vendramim, 2005). En México se siembra alrededor y dentro de los huertos para repeler hormigas, en Argentina y Brasil se coloca en las camas o dentro de las habitaciones para repeler pulgas, y en Camerún algunos productores almacenan su grano mezclado con follaje de esta planta para evitar el daño de plagas (Rodríguez, 2005).

El aceite esencial de Ch. ambrosioides se encuentra en mayor concentración en los frutos y semillas (Gómez, 2008; Monteiro et al., 2017; Langsi et al. 2018), con un rendimiento de aproximadamente $0,02 \%$ de peso seco (Rodríguez, 2005). Sus principales componentes activos son $\alpha$-terpineno, 1,8-cineol, $p$-cimeno, careno y ascaridol (Muhayimana et al., 1998; Onocha et al., 1999; Tapondjou et al., 2002; Gómez, 2008; Chu et al., 2011; Al-kaf et al. 2016), sustancias que podrían ser exploradas como un insecticida natural de contacto (Silva-Aguayo et al., 2005; Tavares y Vendramin, 2005; Langsi et al. 2017), repelente (Novo et al., 1997; Tapondjou et al., 2002; Langsi et al., 2017), y fumigante (Chu et al., 2011; Wei et al., 2015; Bernardes et al., 2018). Por lo descrito, esta investigación tuvo por objetivo evaluar, en condiciones de laboratorio, las propiedades insecticidas e insectistáticas del aceite esencial de follaje e inflorescencia de $\mathrm{Ch}$. ambrosioides contra adultos de $S$. zeamais y su efecto sobre la germinación del maíz.

\section{MATERIALES Y MÉTODOS}

El estudio se realizó en el Laboratorio de Entomología de la Facultad de Agronomía de la Universidad de Concepción, Campus Chillán, Región de Nuble, Chile.

\section{Material vegetal}

La colecta de Ch. ambrosioides se realizó en la comuna de San Fabián $\left(36^{\circ} 33^{\prime} 0^{\prime \prime}\right.$ S, $71^{\circ} 33^{\prime} 0^{\prime \prime}$ W), Región de Nuble, Chile. La recolección se hizo en la etapa de floración, ya que según Tavares y Vendramim (2005) en este estado fenológico se produce la mayor concentración de compuestos químicos secundarios. El aceite esencial se extrajo por hidrodestilación mediante arrastre de vapor con un extractor tipo Clevenger durante dos $h$ (Bittner et al., 2008). Luego, este se depositó en un envase de vidrio color ámbar y se dejó por $24 \mathrm{~h}$ en un freezer a $-15^{\circ} \mathrm{C}$ de temperatura, para extraer el aceite por separación de fases, y posteriormente los residuos restantes de agua se eliminaron con cloruro de calcio anhidro.

El maíz utilizado en los bioensayos y colonias de insectos, correspondió al cultivar Dekalb DK 440 y se obtuvo de la Estación Experimental "El Nogal" de la Facultad de Agronomía de la Universidad de Concepción. Con el objetivo de evitar la alteración de los resultados por alguna infestación previa de insectos o residuo de insecticida, el maíz se lavó con agua potable, se secó a temperatura ambiente por $24 \mathrm{~h}$ y posteriormente se congeló a $-15^{\circ} \mathrm{C}$ por $48 \mathrm{~h}$.

\section{Insectos}

Se utilizaron ejemplares adultos de $S$. zeamais provenientes de colonias permanentes que se mantienen en el Laboratorio de Entomología de la Facultad de Agronomía en frascos de vidrio de $1 \mathrm{~L}$ con maíz como sustrato alimenticio. Estas se mantienen en condiciones controladas de $30 \pm$ $2^{\circ} \mathrm{C}$ de temperatura, $60 \pm 5 \%$ de humedad relativa y oscuridad total en una cámara bioclimática (Memmert Gmbh, IPS 749, Schwabach, Germany). Las colonias se limpiaron cada siete días extrayendo todos los insectos adultos, los que se utilizaron para iniciar nuevas colonias y la 
progenie emergida $\left(\mathrm{F}_{1}\right)$ se ocupó en los bioensayos.

\section{Bioensayos \\ Toxicidad por contacto con grano tratado}

Este bioensayo se realizó con la metodología de Obeng-Ofori y Reichmuth (1997). En envases de vidrio de $500 \mathrm{~mL}$, se mezclaron $100 \mathrm{~g}$ de maíz con una solución de aceite esencial diluido en $1 \mathrm{~mL}$ de acetona, en concentraciones de 0,$25 ; 0,5 ; 1,0 ; 2,0$ y 4,0 \% (v/v), más un testigo consistente en sólo acetona. Los envases se agitaron manualmente por 1 minuto para permitir el cubrimiento uniforme de los granos por la solución, para luego dejarlos a temperatura ambiente por media hora para evaporar la acetona. En seguida, cada frasco se infestó con 10 parejas de insectos, los cuales se diferenciaron por sexo de acuerdo al criterio de Halstead (1963). Posteriormente, los frascos se cubrieron con sus tapas perforadas, para permitir el intercambio gaseoso, y se almacenaron en una cámara bioclimática a $30 \pm 2{ }^{\circ} \mathrm{C}, 60 \pm 5 \%$ de humedad relativa y oscuridad total.

La mortalidad se evaluó a las 24,48 y 72 h y a los 7 y 15 días después de la infestación (DDI), cuantificando los insectos vivos y muertos para obtener el porcentaje de mortalidad que se corrigió con la fórmula de Abbott (1925). Completados los 15 DDI se retiraron todos los insectos de los frascos y se devolvieron a la cámara bioclimática por 40 días más. Finalizado este período (55 DDI) se registró la emergencia de insectos adultos $\left(F_{1}\right)$, contabilizando todos los insectos emergidos después de ese periodo, y considerando como $100 \%$ la $\mathrm{F}_{1}$ del testigo. A los 55 DDI también se evaluó el porcentaje de pérdida de peso mediante la diferencia entre el peso inicial (100 g) y final del grano.

Igualmente a los 55 DDI se realizó una prueba de germinación del maíz con el objetivo de verificar si las diferentes concentraciones de aceite esencial afectan la capacidad de germinación del maíz. Para esto, durante siete días se colocaron a germinar 20 semillas (sin daño aparente) de cada repetición por tratamiento, en placas Petri acondicionadas con un papel filtro húmedo a $25 \pm 2^{\circ} \mathrm{C}$ de temperatura en una cámara bioclimática (Betancur et al., 2010). El porcentaje de germinación se calculó considerando como $100 \%$ el número de semillas germinadas en el testigo.

\section{Toxicidad sobre estados inmaduros $\left(\mathrm{F}_{1}\right)$}

En este bioensayo se usó la metodología de Obeng-Ofori et al. (1998). En envases de vidrio de $500 \mathrm{~mL}$ se colocaron $200 \mathrm{~g}$ de maíz que se infestaron con 10 parejas de insectos adultos de 24 horas de edad, a los que se dejó reproducirse libremente por 21 días. Posteriormente se retiraron los insectos y se mezcló el maíz con una solución de aceite esencial en $1 \mathrm{~mL}$ de acetona, en concentraciones de 0,$25 ; 0,5 ; 1,0 ; 2,0$ y $4,0 \%(\mathrm{v} / \mathrm{v})$, más un testigo consistente en sólo acetona. Durante el experimento los envases se mantuvieron en una cámara bioclimática a una temperatura de 30 $\pm 2^{\circ} \mathrm{C}, 60 \pm 5 \%$ de humedad relativa y oscuridad total. Se evaluó la emergencia de insectos adultos $\left(\mathrm{F}_{1}\right)$ removiendo y contabilizando los insectos emergidos semanalmente durante siete semanas.

\section{Toxicidad por fumigación}

La toxicidad por efecto fumigante se evaluó de acuerdo a la metodología de Chu et al. (2011). En un papel filtro circular de $2,5 \mathrm{~cm}$ de diámetro se aplicó, con una micropipeta, aceite esencial sin diluir en seis concentraciones: 1,$25 ; 2,5 ; 5 ; 10 ; 15$ y $20 \mu \mathrm{L}$ (equivalentes a 8,$3 ; 16,6 ; 33,2 ; 66,4 ; 132,8$ y $265,6 \mu \mathrm{L} \mathrm{L}^{-1}$ aire), más un testigo consistente en sólo el papel filtro. El papel filtro circular se adhirió a la cara interior de la tapa de un envase de $150 \mathrm{~mL}$ que contenía $20 \mathrm{~g}$ de maíz infestado con 10 insectos adultos sin diferenciación de sexo. Estos tratamientos se almacenaron en una cámara bioclimática a $30 \pm 2^{\circ} \mathrm{C}$ de temperatura, 60 $\pm 5 \%$ de humedad relativa y oscuridad total. La mortalidad se evaluó a los 5 DDI, y los resultados se corrigieron con la fórmula de Abbott (1925).

\section{Actividad antialimentaria}

Se utilizó la metodología de Huang et al. (2002) que consistió en elaborar discos con $1 \mathrm{~mL}$ de una solución de $40 \mathrm{~g}$ de harina de trigo en $100 \mathrm{~mL}$ de agua destilada. Sobre estos discos, se distribuyó uniformemente $1 \mathrm{~mL}$ de la solución de aceite esencial en acetona en concentraciones de 0,$25 ; 0,5 ; 1,0 ; 2,0$ y $4,0 \%(\mathrm{v} / \mathrm{v})$, más un testigo consistente en solo acetona. A continuación cada disco se dejó secar por 1 hora a temperatura ambiente para evaporar la acetona. Al mismo tiempo, un grupo de 20 discos se deshidrató en una estufa de convección forzada (Memmert Gmlh, UNB 500, Schwahach, Germany) a $40 \pm$ $5^{\circ} \mathrm{C}$ por $48 \mathrm{~h}$ para obtener el peso seco promedio inicial de los discos.

La preferencia alimentaria de $S$. zeamais se evaluó utilizando placas Petri de $5 \mathrm{~cm}$ de diámetro en las que se colocaron dos discos de harina, uno con la concentración de aceite esencial a evaluar y otro solo con acetona. Luego, cada placa se infestó con 10 insectos adultos de menos de una semana de edad sin diferenciación de sexo, a los que se les permitió alimentarse libremente con el disco de su preferencia durante 5 días. Al final del bioensayo, los restos de los discos de cada repetición se deshidrataron en un horno a $40 \pm 5^{\circ} \mathrm{C}$ por $48 \mathrm{~h}$ para obtener su peso seco, el que se comparó con el peso seco de discos de los discos deshidratados 
al comienzo del bioensayo. Con estos datos se calculó un índice de disuasión de la alimentación IDA $(\%)=(C-T / C+T) * 100$ (Isman et al., 1990), donde $\mathrm{C}$ es la masa ingerida por insectos en discos control (mg) y T es la masa ingerida por insectos en discos tratado (mg). En base al valor del IDA, se utilizaron los criterios de Liu et al., (2007) para clasificar el efecto de los tratamientos como: sin disuasión de la alimentación (IDA $<20 \%$ ), baja disuasión de la alimentación ( $50 \%>$ IDA $\geq 20 \%$ ), moderada disuasión de la alimentación $(70 \%>$ IDA $\geq 50 \%$ ), y fuerte disuasión de la alimentación (IDA $\geq 70 \%$ ).

\section{Repelencia}

La metodología utilizada fue la de Mazzonetto y Vendramim (2003) que consiste en una arena de libre elección formada por cinco placas Petri plásticas de $5 \mathrm{~cm}$ de diámetro y $1,5 \mathrm{~cm}$ de altura, estando una placa central conectada a las otras cuatro por tubos plásticos de $10 \mathrm{~cm}$ de longitud dispuestos diagonalmente formando una " $\mathrm{X}$ ". En envases de vidrio con 50 g de maíz se aplicó $1 \mathrm{~mL}$ de solución de aceite esencial en acetona en concentraciones de 0,$25 ; 0,5 ; 1 ; 2$ y $4 \%(\mathrm{v} / \mathrm{v})$ más un testigo consistente en solo acetona. Luego, los 50 g de maíz de cada concentración se dividieron en partes iguales y se ubicaron en la arena de libre elección en dos placas Petri simétricamente opuestas mientras que en las otras dos placas se ubicó el testigo. En seguida, en la placa central se liberaron 50 insectos adultos sin diferenciación de sexo. Las arenas de libre elección se mantuvieron a temperatura ambiente de $21 \pm 2^{\circ} \mathrm{C}$, y luego de $24 \mathrm{~h}$ se contabilizó el número de insectos por placa. Para la comparación de los tratamientos, se calculó el índice de repelencia (IR) de Procopio et al. (2003) que clasifica un tratamiento como neutro si el índice es igual a 1, atrayente si es mayor a 1 y repelente si es menor a 1, complementado con la escala de Bustos et al. (2017) que de acuerdo al valor de este índice clasifica los tratamientos como: 0,76-0,99 (repelencia débil), 0,51-0,75 (repelencia moderada), 0,26-0,50 (repelencia alta) y $0,0-0,25$ (repelencia muy alta).

\section{Diseño experimental y análisis estadístico}

El diseño experimental fue completamente al azar. Cada tratamiento tuvo cuatro repeticiones y los datos obtenidos se analizaron previamente si cumplían los supuestos de normalidad y homocedasticidad, utilizando las pruebas de Shapiro-Wilks y Levene, respectivamente. Para normalizar los valores porcentuales que no cumplieran con los supuestos, se transformaron a arcoseno $(x / 100)^{1 / 2}$. Luego, los datos se sometieron a un análisis de varianza (ANOVA) y a una prueba de comparación de medias con el test de Tukey $(\alpha \leq 0,05)$. Los análisis se llevaron a cabo con el programa estadístico InfoStat ${ }^{\circledR}$, versión 2008 (Balzarini et al., 2008).

\section{RESULTADOS Y DISCUSIÓN}

\section{Toxicidad por contacto con grano tratado Mortalidad}

La mortalidad obtenida con las distintas concentraciones de aceite esencial fue inferior al $30 \%$, fluctuando entre 0,6 y $25,6 \%$ en las concentraciones de 0,25 y $4 \%$, respectivamente (Tabla 1). Por tanto, se infiere que el aceite esencial de $C h$. ambrosioides no presenta toxicidad por contacto significativa sobre adultos de $S$. zeamais. Estos resultados no concuerdan con Lagsi et al. (2017 y 2018) quienes con una dosis de aceite esencial de Ch. ambrosioides de $200 \mu \mathrm{L}$

Tabla 1. Mortalidad (\%) de adultos de S. zeamais alimentados con grano tratado con diferentes concentraciones de aceite esencial de Chenopodium ambrosioides.

Table 1. Mortality (\%) of S. zeamais adult fed with grain treated with different concentrations of essential oil of Chenopodium ambrosioides.

\begin{tabular}{cccrrr}
\hline Concentración & \multicolumn{5}{c}{ Mortalidad \pm E.E. (\%) } \\
\hline$(\%)$ & $24 \mathrm{~h}$ & $48 \mathrm{~h}$ & $72 \mathrm{~h}$ & 7 días & 15 días \\
\hline 0,25 & $0 \mathrm{a}^{*}$ & $0,0 \pm 0,0 \mathrm{a}$ & $0,0 \pm 0,0 \mathrm{a}$ & $0,0 \pm 0,0 \mathrm{~b}$ & $0,6 \pm 0,0 \mathrm{c}$ \\
0,5 & $0 \mathrm{a}$ & $0,0 \pm 0,0 \mathrm{a}$ & $1,3 \pm 1,3 \mathrm{a}$ & $1,9 \pm 1,1 \mathrm{~b}$ & $11,2 \pm 0,0 \mathrm{~b}$ \\
1,0 & $0 \mathrm{a}$ & $0,0 \pm 0,0 \mathrm{a}$ & $1,3 \pm 1,3 \mathrm{a}$ & $3,8 \pm 0,0 \mathrm{~b}$ & $12,2 \pm 1,5 \mathrm{~b}$ \\
2,0 & $0 \mathrm{a}$ & $1,3 \pm 1,3 \mathrm{a}$ & $3,8 \pm 1,3 \mathrm{a}$ & $5,1 \pm 1,3 \mathrm{~b}$ & $16,4 \pm 3,4 \mathrm{~b}$ \\
4,0 & $0 \mathrm{a}$ & $2,5 \pm 1,4 \mathrm{a}$ & $5,0 \pm 2,0 \mathrm{a}$ & $18,9 \pm 2,1 \mathrm{a}$ & $25,6 \pm 2,1 \mathrm{a}$ \\
\hline C.V. $(\%)$ & - & - & - & 39,9 & 29,7 \\
\hline
\end{tabular}

*Tratamientos con igual letra en la columna no difieren estadísticamente por el test de Tukey $(\alpha=0,05)$; C.V.

$=$ coeficiente de variación; E.E. = error estándar. 
$\mathrm{kg}^{-1}$ obtuvieron una toxicidad entre 80 y $100 \%$ de mortalidad de adultos de $S$. zeamais a las 72 hrs de exposición. Sin embargo, son similares a Kemabonta y Okogbue (2002), quienes utilizando un extracto etanólico de $\mathrm{Ch}$. ambrosioides obtuvieron una mortalidad de 9,1 y $48,2 \%$ con concentraciones de 1,0 y 5,0\%, respectivamente, sobre adultos de Callosobruchus maculatus F. (Coleoptera: Bruchidae). Igualmente Gómez et al. (2018) indican que el follaje pulverizado de esta planta no presenta efecto insecticida de contacto ya que con una concentración de 1,0\% no sobrepasó el $20 \%$ de mortalidad.

No obstante, estudios realizados en Benin, Brasil y Chile, por Procópio et al. (2003), Tavares y Vendramim (2005), Silva-Aguayo et al. (2005), Núñez et al. (2010) y Chougourou et al. (2016), señalan que el polvo de Ch. ambrosioides presenta una mortalidad por contacto entre 69,5 y $100 \%$ con concentraciones de 1,0 y $2,0 \%$ (p/p), respectivamente. Esta diferencia se debería a la variabilidad característica de los derivados vegetales en su concentración de metabolitos secundarios ya que al utilizar polvo la actividad insecticida de los metabolitos secundarios se potencia por la acción física del polvo, que bloquea los espiráculos de los insectos causando una asfixia. Asimismo, el tamaño de las partículas ayuda a una distribución más uniforme del polvo sobre la superficie de las semillas, aumentando la posibilidad de hacer contacto con los insectos (Denloye et al., 2010). En el caso del aceite esencial, como se disuelve en $1 \mathrm{~mL}$ de acetona este no alcanza a cubrir en su totalidad la superficie de los granos, por lo que disminuye la superficie de contacto de los insectos con los compuestos tóxicos, reduciendo su efectividad. Además, cabe destacar que los frascos utilizados, tenían sus tapas perforadas, para permitir el intercambio gaseoso, y al ser el aceite esencial de alta volatilidad, se disipa rápidamente en el ambiente, perdiendo efectividad como insecticida de contacto sobre los insectos tratados.

\section{Emergencia de insectos adultos $\left(F_{1}\right)$}

Los tratamientos se consideraron como promisorios si redujeron al menos un $50 \%$ la $\mathrm{F}_{1}$ de los insectos (Silva-Aguayo et al., 2005). Considerando este umbral, sólo el tratamiento de $4,0 \%$ cumple con el criterio, el cual presentó una $\mathrm{F}_{1}$ de $41,3 \%$ (58,7\% de inhibición) aunque este tratamiento sólo mostró diferencias significativas con la concentración de $0,25 \%$ (Tabla 2). Estos resultados concuerdan con Kemabonta y Okogbue (2002), quienes obtuvieron una emergencia de $48,2 \%$ en adultos de C. maculatus con un extracto etanólico de Ch. ambrosioides a la concentración de 3,0\%. Igualmente Langsi et al. (2018) con una concentración de $200 \mu \mathrm{L} \mathrm{kg}^{-1}$ de aceite esencial de Ch. ambrosioides obtuvieron una inhibición del 100\% de la $\mathrm{F}_{1}$. Además en un estudio realizado un año antes por Langsi et al (2017), en el que mezclaron el aceite esencial de Ch. ambrosioides con el de Cupressus sempervirens en una proporción de 50:50 reportan una inhibición de la progenie de $98 \%$ a partir de la concentración de $100 \mu \mathrm{L} \mathrm{kg}^{-1}$. Sin embargo, resultados de investigación anteriores realizadas por Tavares y Vendramim (2005) contradicen lo mencionado, ya que concluyen que esta planta no afecta la emergencia de adultos de $S$. zeamais. Lo anterior, según Rodríguez (2005) se debe a que algunos ecotipos de $\mathrm{Ch}$. ambrosioides eliminan el total de la población de adultos de $S$. zeamais antes de que ovipositen, y en consecuencia no hay emergencia de insectos en la primera generación,

Tabla 2. Emergencia $\left(\mathrm{F}_{1}\right)$ de $S$. zeamais adultos y pérdida de peso y germinación de maíz tratado con aceite esencial de Chenopodium ambrosioides a los 55 días después de la infestación.

Table 2. Emergence $\left(\mathrm{F}_{1}\right)$ of $S$. zeamais adults and grain weight loss and germination of maize treated with essential oil of Chenopodium ambrosioides at 55 days after infestation.

\begin{tabular}{cccc}
\hline $\begin{array}{c}\text { Concentración } \\
\mathbf{( \% )}\end{array}$ & $\begin{array}{c}\text { Emergencia* } \pm \text { E.E. } \\
(\mathbf{\%})\end{array}$ & $\begin{array}{c}\text { Pérdida de peso* E.E. } \\
\mathbf{( \% )}\end{array}$ & $\begin{array}{c}\text { Germinación } \pm \text { E.E. } \\
\mathbf{( \% )}\end{array}$ \\
\hline 0,00 & -- & $7,7 \pm 0,48 \mathrm{a}$ & -- \\
0,25 & $79,5 \pm 7,1 \mathrm{a}$ & $8,2 \pm 0,85 \mathrm{a}$ & $94,9 \pm 3,3 \mathrm{a}$ \\
0,5 & $65,6 \pm 5,6 \mathrm{ab}$ & $7,5 \pm 1,0 \mathrm{a}$ & $91,0 \pm 3,2 \mathrm{a}$ \\
1,0 & $62,4 \pm 8,1 \mathrm{ab}$ & $7,7 \pm 1,4 \mathrm{a}$ & $97,4 \pm 3,6 \mathrm{a}$ \\
2,0 & $51,6 \pm 5,4 \mathrm{ab}$ & $7,2 \pm 0,25 \mathrm{a}$ & $92,3 \pm 2,1 \mathrm{a}$ \\
4,0 & $41,3 \pm 6,3 \mathrm{~b}$ & $7,0 \pm 0,71 \mathrm{a}$ & $93,6 \pm 2,5 \mathrm{a}$ \\
\hline C.V. $(\%)$ & 21,9 & 22,8 & 6,4
\end{tabular}

*Emergencia del testigo se consideró el 100 \%

** Tratamientos con igual letra en la columna no difieren estadísticamente por el test de Tukey $(\alpha=0,05)$;

C.V.= coeficiente de variación; E.E. = error estándar. 
y en contraste hay otros ecotipos que no matan adultos, sino que reducen la $\mathrm{F}_{1}$ de adultos en la primera generación evitando el daño al grano, siendo esto último concordante con este estudio. Por tanto, el aceite esencial de Ch. ambrosioides suprimiría la oviposición eliminando los huevos y larvas debido a la toxicidad de los compuestos volátiles del aceite esencial (Tapondjou et al., 2002; Denloye et al., 2010).

\section{Pérdida de peso del grano}

Todos los tratamientos mostraron ser estadísticamente similares al testigo. El maíz tratado con aceiteesencial al 2,0 y $4,0 \%$ presentaron los menores porcentajes de pérdida de peso con 7,2 y $7,0 \%$, respectivamente; mientras que la concentración de $0,25 \%$ superó el 8,0\%, siendo incluso superior al testigo (Tabla 2). Los resultados no concuerdan con Dufera et al. (2017) y Gómez et al. (2018) en cuyas investigaciones el polvo $\mathrm{y}$ aceite esencial de Ch. ambrosioides presentaron una pérdida de peso estadísticamente menor que el tratamiento testigo. Igualmente los valores de la presente investigación son mayores a una investigación de mayor data realizada por SilvaAguayo et al. (2005) cuyos valores de pérdida de peso fueron inferiores a 3,0\% con maíz tratado con polvo de Ch. ambrosioides. La diferencia entre los resultados y los antecedentes antes mencionados se debe a que la toxicidad por contacto del polvo de $\mathrm{Ch}$. ambrosioides reportada por estos autores fue mayor a lo logrado en esta investigación, alcanzando una mortalidad de 37,5 y $69,4 \%$ y una $\mathrm{F}_{1}$ de $15,7 \%$ (84,3\% de inhibición) y $14,2 \%(85,8 \%$ de inhibición), respectivamente, por lo que había menor cantidad de insectos sobrevivientes y de la $\mathrm{F}_{1}$. Además todo el desarrollo larval de $S$. zeamais ocurre en el interior de la semilla, alimentándose de esta, por lo que es razonable que con una menor mortalidad y mayor emergencia de adultos, sea mayor la pérdida de peso.

\section{Germinación del maíz}

La germinación del maíz tratado con aceite esencial no presentó diferencias estadísticas entre tratamientos, registrándose valores entre 91,0 y $97,4 \%$ (Tabla 2). Similares resultados obtuvieron Dufera et al. (2017) y Gómez et al. (2018) quienes concluyeron que la planta completa deshidratada y pulverizada de $\mathrm{Ch}$. ambrosioides mantiene la calidad y no afecta el porcentaje de germinación de las semillas de maíz hasta por 150 días. Igualmente estos resultados concuerdan con estudios anteriores realizados en Chile por SilvaAguayo et al. (2005) y Núñez et al. (2010) quienes reportaron que el polvo de $\mathrm{Ch}$. ambrosioides no afecta significativamente el poder germinativo de los granos. Además, la germinación obtenida en la presente investigación (mayor a 85\%) cumple con los porcentajes de germinación establecidos por los estándares nacionales para ser comercializadas como semilla (SAG, 2000).

\section{Toxicidad sobre estados inmaduros $\left(\mathrm{F}_{1}\right)$}

La concentración de aceite esencial de $4,0 \%$ presentó a la séptima semana el menor porcentaje de emergencia de insectos adultos $\left(\mathrm{F}_{1}\right)$ con $47,9 \%$ (52,1\% de inhibición), difiriendo significativamente de los restantes tratamientos que superaron el $60 \%$ de $\mathrm{F}_{1}$ en relación al testigo (Tabla 3). Además, 4,0\% es el único tratamiento que cumple con el criterio propuesto por SilvaAguayo et al. (2005) para ser considerado como promisorio al inhibir el 50\% de la emergencia de insectos adultos. Las concentraciones de 1,0 y $2,0 \%$ alcanzaron una emergencia de 77,5

Tabla 3. Emergencia $\left(\mathrm{F}_{1}\right)$ de adultos de $S$. zeamais en maíz tratado con diferentes concentraciones de aceite esencial de $C h$. ambrosioides.

Table 3. Emergence $\left(\mathrm{F}_{1}\right)$ of $S$. zeamais adults treated with different concentrations of essential oil of Chenopodium ambrosioides.

\begin{tabular}{cccc}
\hline $\begin{array}{c}\text { Concentración } \\
\text { (\%) }\end{array}$ & \multicolumn{3}{c}{$\begin{array}{c}\text { Emergencia (número de insectos)* } \\
\text { Semana }\end{array}$} \\
\cline { 2 - 4 } & (21 DDI) & (35 DDI) & Semana 7 \\
\hline 0,25 & $0,0 \mathrm{a}^{*}$ & $81,6 \pm 7,5 \mathrm{a}$ & $92,9 \pm 5,4 \mathrm{a}$ \\
0,5 & $0,0 \mathrm{a}$ & $73,5 \pm 6,7 \mathrm{ab}$ & $94,4 \pm 7,4 \mathrm{a}$ \\
1,0 & $0,0 \mathrm{a}$ & $53,1 \pm 12,6 \mathrm{ab}$ & $77,5 \pm 15,1 \mathrm{ab}$ \\
2,0 & $0,0 \mathrm{a}$ & $46,9 \pm 3,9 \mathrm{ab}$ & $63,4 \pm 8,1 \mathrm{ab}$ \\
4,0 & $0,0 \mathrm{a}$ & $38,8 \pm 8,4 \mathrm{~b}$ & $47,9 \pm 5,4 \mathrm{~b}$ \\
\hline C.V. $(\%)$ & - & 28,4 & 24,0
\end{tabular}

*Tratamiento con igual letra en la columna no difieren estadísticamente por el test de Tukey $(\alpha=0,05)$

** DDI = Días después de infestación; C.V. = coeficiente de variación; E.E. = error estándar. 
y $63,4 \%$, respectivamente, mientras que las concentraciones de 0,25 y 0,5\% superaron el $90 \%$ de emergencia de adultos de S. zeamais sin diferir estadísticamente del testigo. Con base a los resultados obtenidos, se puede señalar que el efecto insecticida sobre estados inmaduros del aceite esencial de Ch. ambrosioides se produce solo en concentraciones iguales o superiores a $4,0 \%$. Igualmente, se observó que la emergencia de $S$. zeamais se produce 30 días después de la oviposición, lo que concuerda con Betancur et al. (2010) por lo que el control de los estados inmaduros se debería hacer durante este periodo.

\section{Efecto fumigante}

El aceite esencial de Ch. ambrosioides presentó actividad fumigante significativa ya que en todos los tratamientos evaluados se logró un 100\% de mortalidad de adultos de S. zeamais. Estos resultados concuerdan con Bernardes et al. (2018) quienes con una concentración de $500 \mu \mathrm{L} \mathrm{L}^{-1}$ aire, casi el doble que la máxima concentración evaluada en la presente investigación, obtuvieron un $100 \%$ de mortalidad. Además, Chu et al. (2011) quienes también obtuvieron un 100\% de mortalidad determinaron que Ascaridol es el compuesto del aceite esencial de Ch. ambrosioides que presenta la mayor actividad fumigante contra adultos de $S$. zeamais. Por otra parte, el aceite esencial de Ch. ambrosioides también ha presentado una alta eficacia como fumigante contra otras especies de insectos asociados a cereales almacenados. Al respecto Bernardes et al. (2018) registraron una toxicidad de $100 \%$ sobre Zabrotes subsfaciatus (Coleoptera: Bruchidae). Igualmente en investigaciones anteriores Tapondjou et al. (2002) reportaron una mortalidad superior a $80 \%$ de Acanthoscelides obtectus Say, Callosobruchus chinensis L. (Coleoptera: Bruchidae), Sithopilus granarius L. (Coleóptera: Curculionidae) y Prostephanus truncatus Horn
(Coleóptera: Bostrichidae) con una dosis de aceite esencial de Ch. ambrosioides de $0,2 \mu \mathrm{L} \mathrm{cm}$ ${ }^{2}$. En contraste, en esta misma investigación se determinó que, entre los insectos evaluados, $S$. zeamais es el menos susceptible al aceite esencial, alcanzando una mortalidad entre 5 y $20 \%$ con dosis de 0,2 y $0,8 \mu \mathrm{L} \mathrm{cm}^{-2}$ después de $24 \mathrm{~h}$ de exposición. Igualmente, Denloye et al. (2010) utilizando aceite esencial de esta misma planta obtuvieron mayor toxicidad como fumigante contra C. maculatus que $S$. zeamais, hecho que no concuerda con esta investigación, que muestra una mortalidad del $100 \%$ de S. zeamais. Estos últimos antecedentes son relevantes, debido a que en esta investigación se eliminó con todas las concentraciones evaluadas la totalidad de los adultos de $S$. zeamais considerada la especie menos susceptible, lo cual confirma el potencial del aceite esencial de $C h$. ambrosioides como una alternativa para el control de plagas de granos almacenados.

\section{Actividad antialimentaria}

El único tratamiento, de acuerdo a los criterios de Liu et al. (2007), que no presentó disuasión de la alimentación fue la concentración de $0,25 \%$, con un IDA de $-50 \%$ (Tabla 4). El valor negativo indica que el consumo de los insectos fue mayor en el disco impregnado con esta concentración de aceite esencial que en el testigo. Todos los tratamientos restantes mostraron un IDA igual o mayor al $20 \%$, aunque menores a $50 \%$, lo cual, según los mismos autores, clasifica a los tratamientos como con baja disuasión de la alimentación. La limitada disuasión de la alimentación puede deberse a que el compuesto que evita que el insecto responda negativamente al estímulo gustativo se encuentra en baja concentración (Rodríguez et al., 2003). Además el efecto antialimentario del aceite esencial de Ch. ambrosioides es menor que otras especies vegetales como Schinus molle

Tabla 4. Índice de disuasión de la alimentación (IDA) de S. zeamais para diferentes concentraciones de aceite esencial de Chenopodium ambrosioides.

Table 4. Dissuasive alimentary index (IDA) of S. zeamais for different concentrations of essential oil of Chenopodium ambrosioides.

\begin{tabular}{ccc}
\hline Concentración & IDA $(\boldsymbol{\%})$ & Efecto $^{*}$ \\
\hline 0,25 & $-50,0$ & SDA \\
0,5 & 43,1 & BDA \\
1,0 & 49,2 & BDA \\
2,0 & 43,7 & BDA \\
4,0 & 49,4 & BDA \\
\hline
\end{tabular}

*Sin disuasión de la alimentación (SDA) (IDA < 20\%); baja disuasión de la alimentación (BDA) $(50 \%>$ IDA $\geq 20 \%)$; moderada disuasión de la alimentación (MDA) $(70 \%>$ IDA $\geq$ $50 \%$ ); fuerte disuasión de la alimentación (FDA) (IDA $\geq 70 \%$ ) (Liu et al., 2007). 
L. var. Areira (Anacardiaceae) y Myracrodruon urundeuva Engl. (Anacardiaceae) (Benzi et al., 2009), las cuales obtuvieron efectos disuasivos moderados de 62 y $60 \%$ en adultos de $S$. oryzae y $S$. zeamais, respectivamente. Sin embargo se debe destacar que el aceite esencial de $\mathrm{Ch}$. ambrosioides ha presentado efecto antialimentario contra otras especies de insectos como Plutella xylostella (Lepidoptera: Iponomeutidae) cuyas larvas de tercer estadio disminuyen el consumo foliar en la medida que se incrementa la dosis de aceite esencial (Wei et al., 2015). Igualmente Trindade et al (2015) obtuvieron que larvas de Spodoptera frugiperda (Lepidoptera: Noctuidae) alimentadas con hojas tratadas con aceite esencial de $\mathrm{Ch}$. ambrosioides comen menos disminuyendo el peso y tamaño de la pupa.

\section{Repelencia}

El único tratamiento que no mostró efecto repelente fue la concentración de 0,25\% (Tabla 5), clasificándose como neutro de acuerdo al IR de Procopio et al. (2003). Todos los restantes tratamientos mostraron IR menores a 1, lo cual los clasifica como repelentes. Sin embargo, el mayor efecto se obtuvo con las concentraciones de 2,0 y $4,0 \%$ con un IR de 0,49 y 0,31 que según Bustos et al. (2017) se clasifican como con repelencia alta coincidiendo con Langsi et al. (2017) quienes señalan al aceite esencial de esta especie como altamente repelente contra adultos de $S$. zeamais. Igualmente Bernardes et al. (2018) indican que una concentración de 500 $\mu \mathrm{L} \mathrm{kg}^{-1}$ concluyen que el aceite esencial de Ch. ambrosioides muestra actividad repelente eficiente contra adultos de Z. subfasciatus plaga primaria de frejol almacenado. Además, el polvo de $C h$. ambrosioides en concentraciones de 1,0; 2,0 y $3,0 \%(\mathrm{p} / \mathrm{p})$ ha mostrado valores similares a la presente investigación (Silva-Aguayo et al. 2005, Mazzonetto et al. 2013, Gómez et al. 2018).

Sin embargo, los resultados difieren de Tavares y Vendramim (2005) y Procópio et al., (2003), quienes determinaron que el polvo de Ch. ambrosioides no es repelente para $S$. zeamais a una concentración de 3,0 \% (p/p). Este hecho podría explicarse en que Tavares y Vendramim (2005), utilizaron frutos, tallos y hojas, y Procópio et al., (2003) flores, frutos y hojas, mientras que en el presente bioensayo se ocupó solo la inflorescencia, la cual de acuerdo a Rodríguez (2005) concentra la mayor cantidad de compuestos secundarios. Cabe destacar que el aceite esencial de $C h$. ambrosiodes también ha mostrado actividad repelente contra mosquitos como Aedes aegyti (Diptera: Culicidae) (Azeem et al. 2019) y Anopheles gambiae (Diptera: Culicidae) (Oumarou et al. 2018) y ácaros fitófagos como Tetranychus urticae (Acari: Tetranychidae) (Musa et al. 2017). Finalmente, se puede señalar que los resultados obtenidos en la presente investigación son de gran utilidad, para considerar al aceite esencial de Ch. ambrosioides como una alternativa efectiva para el control de S. zeamais; sin embargo, estos deben ser evaluados y validados a mayor escala en condiciones de bodega.

\section{CONCLUSIONES}

Los resultados obtenidos permiten concluir que el aceite esencial de Ch. ambrosioides en condiciones de laboratorio presenta actividad insecticida fumigante e insectistática como repelente y antialimentario contra adultos de $S$. zeamais, sin afectar la germinación de semillas de maíz.

\section{LITERATURA CITADA}

Abbott, W.S. 1925. A method of computing the effectiveness of an insecticide. J. Econ. Entomol. 18(2):265-267.

Al-kaf, A.G., R. Crouch, A. Denkert, A. Porzel, S.S. Al-Hawshabi, N.A. Ali, et al. 2016. Chemical composition and biological activity of essential oil of Chenopodium ambrosioides from Yemen. Am. J. Essent. Oil. Nat. 4(1):2022.

Tabla 5. Repelencia del aceite esencial de Chenopodium ambrosioides sobre adultos de S. zeamais. Table 5. Repellency of essential oil of Chenopodium ambrosioides against S. zeamais adults.

\begin{tabular}{ccl}
\hline Concentración (\%) & Índice de repelencia (IR) & Efecto $^{*}$ \\
\hline 0,25 & 1,00 & Neutro \\
0,5 & 0,85 & Repelente \\
1,0 & 0,88 & Repelente \\
2,0 & 0,49 & Repelente \\
4,0 & 0,31 & Repelente \\
\hline
\end{tabular}

${ }^{*} \mathrm{IR}>1$ atrayente; IR = 1 neutro; IR $<1$ repelente (Mazzonetto y Vendramim, 2003). 
Azeem, M., T. Zaman, M. Tahir, A. Harris, Z. Iqbal, M. Binyameen, et al. 2019. Chemical composition and repellent activity of native plants essential oils against dengue mosquito, Aedes aegypti. Ind. Crop Prod. 140:1-7

Balzarini, M.G., L. González, M. Tablada, F. Casanoves, J.A. Di Rienzo, y C.W. Robledo. 2008. InfoStat: software estadístico. Manual del usuario. Versión 2008. Brujas, Córdoba, Argentina.

Benzi, V., N. Stefanazzi, and A.A. Ferrero. 2009. Biological activity of essential oils from leaves and fruits of pepper tree (Schinus molle L.) to control rice weevil (Sitophilus oryzae L.). Chilean J. Agric. Res. 69(2):154-159.

Bernardes, W., E. Silva, A. Crotti, and E. Baldin. 2018. Bioactivity of selected plant-derived essential oil against Zabrotes subfaciatus (Coleoptera: Bruchida). J. Stored Prod. Res. 77(1):16-19.

Betancur, J., G. Silva, J.C. Rodríguez, S. Fischer, and N. Zapata. 2010. Insecticidal activity of Peumus boldus Molina essential oil against Sitophilus zeamais Motschulsky. Chilean J. Agric. Res. 70(3):399-407.

Bittner, M.L., M.E. Casanueva, C.C. Arbert, M.A. Aguilera, V.J. Hernández, and J.V. Becerra. 2008. Effects of essential oils from five plant species against the granary weevils Sitophilus zeamais and Acanthoscelides obtectus (Coleóptera). J. Chilean Chem. Soc. 53(1):1455-1459.

Bustos, G., G. Silva, S. Fisher, I. Figueroa, A. Urbina, y J.C. Rodríguez. 2017. Repelencia de mezclas de aceites esenciales de boldo, laurel chileno y tepa contra el gorgojo del maíz. Southwest Entomol. 42(2):551-562.

Chu, S.S., J.F. Hu, and Z.L. Liu. 2011. Composition of essential oil of Chinese Chenopodium ambrosioides and insecticidal activity against maize weevil, Sitophilus zeamais. Pest Manag. Sci. 67(6):714-718.

Chougourou, D., C. Agossa, Y. B. Zoclanclounon, M.G. Nassara, and A. Agbaka. 2016. Efficacy of two plants powders as cowpea grain protectans against Callosobruchus maculatus Fabricius (Coleoptera, Chrysomelidae:Bruchinae). J. Appl. Biosci. 105:10152-10156.

Danho, M., C. Gaspar, and E. Haubruge. 2002. The impact of grain quantity on the biology of Sitophilus zeamais Motschulsky (Coleoptera: Curculionidae): oviposition, distribution of eggs, adult emergence, body weight and sex ratio. J. Stored Prod. Res. 38(3):259-266.
Denloye, A.A., W.A. Makanjuola, O.K. Teslim, O.A. Alafia, A.A. Kasali, and A.O. Eshilokun. 2010. Toxicity of Chenopodium ambrosioides L. (Chenopodiaceae) products from Nigeria against three storage insects. J. Plant Prot. Res. 50(3):379-384.

Dufera, L., A. Tadesse, W. Gobena, and C. Kuyu. 2019. On farm evaluation of Eucalyptus globulus Labill leaf and Chenopodium ambrosioides L. whole plant powder against storage insect pests in stored maize at Sokoru district in Jimma zone of Oromia regional state, Ethiopia. Psyche. doi:10.11648/j. aff.20130205.13.

Gómez, J.R. 2008. Epazote (Chenopodium ambrosioides). Revisión a sus características morfológicas, actividad farmacológica, y biogénesis de su principal principio activo, ascaridol. Bol. Latinoam. Caribe Plantas Med. Aromát. 7(1):3-9.

Gómez, J.R., O. González and J.C. González. 2018. Vegetales pulverizados para el manejo de Sitophilus zeamais Motschulsky en almacenamiento. Rev. Mex. Cienc. Agr. 9(4):787-798.

Halstead, D.G.H. 1963. External sex differences in stored-products Coleoptera. Bull. Entomol. Res. 54(1):119-134.

Huang, Y., S.-H. Ho, H.-C. Lee, and Y.-L. Yap. 2002. Insecticidal properties of eugenol, isoeugenol and methyleugenol and their effects on nutrition of Sitophilus zeamais Motsch. (Coleoptera: Curculionidae) and Tribolium castaneum (Herbst) (Coleoptera: Tenebrionidae). J. Stored Prod. Res. 38(5):403412.

Isman, M.B. 2015. A renaissance for botanical insecticides?. Pes. Manag. Sci. 71:1587-1590.

Kemabonta, K.A., and F. Okogbue. 2002. Chenopodium ambrosioides (Chenopodiaceae) as a grain protectant for the control of the cowpea pest Callosobruchus maculatus (Coleoptera Bruchidae). J. Fruit Ornam. Plant Res. 10:165-171.

Langsi, D.J., C.N. Fokunang, C. Sun, and W.J. Goudoungou. 2017. Potential of essential oils of Chenopodium ambrosioides L. and Cupressuss sempervirens L. against stored maize pest, Sitophilus zeamais. Motschulsky. J. Entomol. Zool. Stud. 5(2):309-313.

Langsi, D.J., H.K. Tofel, C.N. Fokunang, C. Sun, K. Eloh, P. Caboni, and E.N. Nukenini. 2018. Insecticidal activity of essential oils of Chenopodium ambrosioides L. and Cupressuss sempervirens L. ad their binary combinations on Sitophilus zeamais. GSC Biological and Pharmaceutical Sciences 3(2):24-34. 
Liu, Z.L., S.H. Goh, and S.H. Ho. 2007. Screening of Chinese medicinal herbs for bioactivity against Sitophilus zeamais Motschulsky and Tribolium castaneum (Herbst). J. Stored Prod. Res. 43(3):290-296.

Mazzonetto, F., e J.D. Vendramim. 2003. Efeito de pós de origem vegetal sobre Acanthoscelides obtectus (Say) (Coleoptera: Bruchidae) em feijão armazenado. Neotrop. Entomol. 32(1):145-149.

Mazzonetto, F., R. Zapparoli, and A. Dalri. 2013. Biofumigation of plant species on Sitophilus zeamais in stored maize. Applied Research \& Agrotecnology 6(1):53-61.

Monteiro, J.N., A. Archanjo, G. Passos, A. Costa, L. Porfirio, and I. Freire. 2017. Chenopodium ambrosioides L. essential oil and ethanol extract on control of canine Ancylostoma spp. Ciencias Agrarias 38(4):1947-1954.

Muhayimana, A., J.-C. Chalchat, and R.P. Garry. 1998. Chemical composition of essential oils of Chenopodium ambrosioides L. from Rwanda. J. Essent. Oil Res. 10(6):690-692.

Musa, A., I. Medo, I. Maric, and D. Marcic. 2017. Acaricidal and sublethal effects of a Chenopodium-based biopesticide on the twospotted spider mite (Acari: Tetranychidae). Exp. Appl. Acarol. 71:211-226.

Novo, R.J., A. Viglianco, y M. Nassetta. 1997. Actividad repelente de diferentes extractos vegetales sobre Tribolium castaneum (Herbst). Agriscientia 14:31-36.

Núñez, P., G. Silva, M. Tapia, R. Hepp, J.C. Rodríguez-Maciel, y A. Lagunes-Tejeda. 2010. Toxicidad de polvos de follaje de paico (Chenopodium ambrosioides L.) y boldo (Peumus boldus Mol.) solos y en mezcla con carbonato de calcio sobre gorgojo del maíz (Sitophilus zeamais Motschulsky). AgroCiencia 26(2):71-80.

Obeng-Ofori, D., and C. Reichmuth. 1997. Bioactivity of eugenol, a major component of essential oil of Ocimum suave (Wild.) against four species of stored-product Coleoptera. Int. J. Pest Manag. 43(1):89-94.

Obeng-Ofori, D., C.H. Reichmuth, A.J. Bekele, and A. Hassanali. 1998. Toxicity and protectant potential of camphor, a major component of essential oil of Ocimum kilimandscharicum, against four stored product beetles. Int. J. Pest Manag. 44(4):203-209.

Onocha, P.A., O. Ekundayo, T. Eramo, and I. Laakso. 1999. Essential oil constituents of Chenopodium ambrosioides L. leaves from Nigeria. J. Essent. Oil Res. 11(2):220-222.
Oumarou, M., L. Younoussa and E. Nukenine. 2018. Toxic effct of Chenopodium ambrosioides, Hyptis suaveolens and Lippia adoensis leaf metanol extracts and essential oils against four instar larvae of Anopheles gambiae (Diptera: Culicidae). Int. J. Mosq. Res. 5(1):6166.

Procópio, S., J. Vendramim, J. Ribeiro, e J. dos Santos. 2003. Bioatividade de diversos pós de origem vegetal em relaçao a Sitophilus zeamais Mots. (Coleoptera: Curculionidae). Ciênc. Agrotecnol. 27(6):1231-1236.

Rodríguez, C., G. Silva, y J. D. Vendramim. 2003. Insecticidas de origen vegetal. p. 8791. En G. Rodríguez, C. 2005. Plantas contra plagas 2. Epazote, hierba de la cucaracha, paraíso, higuerilla y sabadilla. Colegio de Postgraduados, Montecillo, México.

SAG (Chile). 2000. Normas generales y específicas de certificación de semillas. Disponible en Universidad Austral de Chile. <http:// fitotecnia.uach.cl/manejador/resources/ normasgeneralessemillas.pdf $>$. (Consulta: 10 mayo 2015)

Sarwar, M. 2015. The killer chemicals for control of agricultura insect pests: the botanical insecticides. International Journal of Chemical and Biomolecular Science 1(3):123128.

Silva-Aguayo, G.I., R. Kiger-Melivilu, R. HeppGallo, y M. Tapia-Vargas. 2005. Control de Sitophilus zeamais con polvos vegetales de tres especies del genero Chenopodium. Pesq. Agropec. Bras. 40(10):953-960.

Silva, G, y R. Hepp (eds.). Bases para el manejo racional de insecticidas. Universidad de Concepción. Facultad de Agronomía / Fundación para la Innovación Agraria, Chillán, Chile.

Suthisut, D., P.G. Fields, and A. Chandrapatya. 2011. Contact toxicity, feeding reduction, and repellency of essential oils from three plants from the ginger family (Zingiberaceae) and their major components against Sitophilus zeamais and Tribolium castaneum. J. Econ. Entomol. 104(4):1445-1454.

Tapondjou, L.A., C. Adler, H. Bouda, and D.A. Fontem. 2002. Efficacy of powder and essential oil from Chenopodium ambrosioides leaves as post-harvest grain protectants against six-stored products beetles. J. Stored Prod. Res. 38(4):395-402.

Tavares, M.A.G.C., e J.D. Vendramim. 2005. Bioatividade da erva-de-Santa-Maria, Chenopodium ambrosioides L., sobre Sitophilus zeamais Mots. (Coleoptera: Curculionidae). Neotrop. Entomol. 34(2): 319-323. 
Trindade, R.C.P., E.S. Ferreira, I.B. Gomes, L. Silva, A.E.G. Santána, S.M.F. Broglio, et al. 2015. Extratos aquosos de inhame (Dioscorea rotundata Poirr.) e de mastruz (Chenopodium ambrosioides L.) no desenvolvimento da lagarta-do-cartucho-do-milho Spodoptera frugiperda (J.E. Smith, 1797). Rev. Bras. Pl. Med. 17(2)291-296.

Wei, H., J. Liu, B. Li, Z. Zhan, Y. Chen, H. Tian, S. Lin, and X. Gu. 2015. The toxicity and physiological effect of essential oil of Chenopodium ambrosioides against the diamonback moth, Plutella xylostella (Lepidoptera: Plutellidae). Crop Prot. 76:6874. 